Manlio Cortelazzo

Padooa

\title{
VOCI ZINGARE NEI GERGHI PADANI
}

In quale misura la lingua zingara ha contribuito a costituire ed alimentare l'eterogeneo lessico gergale? Piuttosto scarsa, verrebbe d'arguire, scorrendo l'unico studio rigoroso e sistematico sull'argomento: delle centinaia di voci (1400) attinte direttamente da A. Menarini alle parlate degli emarginati bolognesi, solo diciannove sono di sicura o molto probabile origine zingara (di cui nove, le prime dell'elenco, mai segnalate prima). Il reperimento, condotto con rara competenza ed abbondanza di riscontri da C. Tagliavini e dallo stesso Menarini, riguarda le seguenti parole:

1. bó/n(i)a, bó/ma s. f. 'donna di servizio' < busnó 'persona estranea, non zingara';

2. čai s. m. 'contadino' < čavo 'figlio' incrociato con čai 'figlia';

3. kakaña s. f. 'gallina'< khakéna 'id.';

4. karamaska s. f. 'pistola' < karamàskre 'id.';

5. királ s. m. 'formaggio' < kiral, keral 'id.';

6. lampo s. m. 'olio' < (zing. sp.) ampio 'id.';

7. lāoo s. m. 'nome' < lav 'parola', 'nome';

8. stardù p. p. 'arrestato < (zing. it.) (e)shtardó 'id.';

9. zént s. m. 'girovago', 'gergo' < sinto 'zingaro';

10. čóll, čullén s. m. 'coltello da tasca' < čuri, (zing. sp.) chulo 'id.';

11. čukél s. m. 'cane' < ğukel 'id.';

12. čuribé s. m. 'ladro' < čoribe(n) 'furto';

13. gağo s. m. 'contadino, sempliciotto' < găğo 'estraneo, non zingaro';

14. górum s. m. 'vecchio' < gōrō 'estraneo sposato con una zingara';

15. grai, krai s. m. 'cavallo' < grai 'id.';

16. lóvi s. m. 'soldi, denaro' < (zing. it.) lovi 'id.';

17. marók s. m. 'pane'< (zing. it.) maro 'id.';

18. stárebi s. m. 'prigione' < (zing. it.) starbè 'id.';

19. pióla s. f. 'negozio, osteria' < (zing. it.) piela 'egli beve'.

Questa limitazione quantitativa dell' apporto zingarico confermerebbe l'opinione espressa dal Dauzat (Les Argots 85) sull'insignificante influenza degli Zingari - poco numerosi e sempre erranti - nella vita e nella 


\section{Linguistica $X V$}

parlata dei malfattori, ma sarebbe in contrasto con le conclusioni, alle quali è giunto il Wagner a proposito del gergo di Barcellona, in cui quasi la metà dei vocaboli sarebbe di origine zingarica.

Un correttivo alla considerazione eccessivamente ristrettiva dell'elemento zingaro nei gerghi italiani (già sostenuta dal Miklosich) è stato proposto dal medesimo Wagner e, sulla sua scia, dal Pellis, esaminando il ricchissimo, ma non sempre sicuro materiale raccolto agli inizi del secolo dal Mirabella nel domicilio coatto dell'isoletta trapanese di Favignana. Sono parecchie voci, che aumenterebbero l'esile lista bolognese (la quale, tuttavia, appagava il raccoglitore: "gli zingarismi entrano nel furbesco bolognese in misura abbastanza sensibile», A. Menarini, I gerghi bolognesi 14), se si potesse essere certi della loro origine, diffusione e larga conoscenza: non manca, infatti, il sospetto, che alcuni informatori siano stati veri e propri zingari.

Per contribuire alla soluzione del problema (scarsità o abbondanza di voci zingare nei gerghi della malavita italiana?) presentiamo alcuni elementi, ${ }^{1}$ tratti da fonti più prossime a noi (l'ultimo trentennio) e limitate alla pianura padana: al Veneto (Padova e Verona) e alla bassa lombarda (in seguito citate come crem. $=$ cremonese). ${ }^{2}$

Notiamo, innanzitutto, come gli zingarismi registrati a Bologna siano, in parte, di più largo e comune impiego:

1. čai s. m. 'contadino, paesano': pad. (Cir. 227) e ver. (Sol. 12); cfr. nel gergo turco çay col significato, aderente all'originale, di 'ragazzo' (Kostov 85);

2. kakaña s. f. 'gallina': pad. (kalkaña Cald. 192) e ver. (Sol. 10); 'pollo': crem. (Mont. gloss.);

3. karamà(s)kari s.f. 'rivoltella': pad. (Miss. 29, Cald. 192; anche karamàngari Cald. 192 e, per accostamento ad un nome femminile abbastanza comune, Màlgari 'Margherita', cara malgari Agno e caramalgari Cir. 231), ver. (Sol. 12) e crem. (calamastra Mont. gloss.); le due varianti

1 Particolarmente per le voci attestate da un'unica fonte deve valere la prudente osservazione di Tagl:-Men. 245 n. 2: «la presenza di una voce zingara isolata non può provare che essa sia veramente entrata nel gergo; solo quand'essa ricorre in frasi gergali generalmente usate si può parlare di voce mutuata dallo zingaro». Da parte nostra possiamo aggiungere che, per esempio, /minči sùkari 'bella ragazza' era già stato da noi registrato nel 1940 nel corso di un colloquio con alcuni gerganti veronesi.

2 Desidero rinnovare pubblicamente il mio ringraziamento più vivo alla dott. Emanuela Caldognetto Magno, che ha messo liberalmente a mia disposizione non solo la sua tesi di laurea (nella quale, in successiva elaborazione, aveva già individuata l'origine zingara delle voci gergali padovane da lei direttamente raccolte), ma anche i risultati inediti di inchieste svolte a Padova, interrogando alcune zingare e un ambulante. - Sono, inoltre, debitore all'amichevole cortesia dei professori M. Pfister (Saarbrücken), R. Vidović (Spalato) e G. Soravia (Milano), che hanno prontamente aderito alla mia richiesta, procurandomi alcuni saggi fondamentali di difficile accesso, ed il secondo anche preziosi suggerimenti (cit. con: comunic. Soravia). 
risalgono al sing. (karamáskeri) e al pl. (karamángeri) rispettivamente [comunic. Soravia]);

4. stàrdelo v. 'uccidilo con violenza': ver. (Sol. 38 , letteralmente e originariamente col probabile significato di 'préndilo');

5. sinto s. m. 'zingaro': pad. (Cir. 237, al pl.), ver. (Sol. 36) e crem. (Mont. 395: «con lui ebbi affari durante il periodo che trascorsi coi 'sinti's):

6. čuri s. m. 'coltello': pad. (Cald. 190, anche čurli) e ver. (Sol. 13); ampiamente diffuso anche negli altri gerghi, dal franc. (Esnault 129-130) al serbo-croato (Uhlik 12), al turco (Kostov 86-87);

7. čukke s. m. 'cane': pad. (Cald. 190) e ver. (ğùkerle Andr. 413, giùcarle Sol. 19); cfr. Uhlik 14 per il serbo-croato;

8. gaîo s. m. 'persona che può essere facilmente raggirata': pad. (Cald. 191, penetrato anche nel dialetto: gàio 'contadino', come nel trevis. gağo 'contadino', leggermente spregiativo: G. Màfera in «Atti Ist. Ven.» CXXX, 1971 - 72, p. 78), ver. (gàgio 'contadino, paesano' Sol. 17) e crem. (gaggio 'uomo' Mont gloss.); diffuso un po' dovunque (per il gergo franc. v. Esnault 143 e Max 16);

9. grai s. m. 'cavallo': pad. (Cir. 229, Cald. 191), ver (Sol. 19) e crem. (Mont. gloss., Mont. 353: «il padre Caleandro era in carcere per ricettazione di grai (cavalli)», Mont. 384: «noi ... si stava slegando il 'grai' (cavallo)»);

10. maròko s. m. 'pane': pad. (Agno, Cir. 232, Cald. 193), ver. (Andr. 415, Sol. 23) e crem. (maròch Mont. gloss., Mont. 427: «buta la sboba ed il maroch tira a campà»);

11. starape s. f. 'carcere': crem. (Mont. 350: «da pochi giorni era uscito da Starape (carcere)》) e con vari fraintendimenti anche ver. (stèri bèi Andr. 418, stari-bèi Sol. 38); la voce zingarica - raccolta dal Pellis n. 2624: v. le aggiunte di G. Prati, da Partisani in Piemonte: starebén, e in Lombardia: stàrepe, stàrebe - è stata divisa in due segmenti, riconoscendo nel secondo un agg. e traendo di conseguenza il s. m. stari, steri, vivo tanto nel ver. (Andr. 418, Sol. 38), quanto nel pad. (stari Cir. 238, Cald. 198); per la sua presenza in altri gerghi, cfr. il franc. staripen (Esnault 135), chtar (Max 16, dove si tende a dar credito all'insostenibile derivazione da štar 'quattro' (sottinteso mura), decisamente respinta dal Partesani), il ted. Stariben (Wolf Rotro. n. 5553), ecc.;

12. piòla s. f. 'bottega in genere, bar, osteria': pad. (Cir. 234, Cald. 195), ver. (Sol. 29) e crem. (Mont. gloss.). - Alla stessa base pi- 'bere' si potrebbe ricondurre tutta una serie di voci strettamente legate al medesimo concetto, fatte salve le riserve su eventuali collisioni ed incroci già poste in chiaro da Tagl.-Men. 278-280: crem. pimes s. m. pl. 'ubriachi' (Mont. gloss., Mont. 355: «finché venne mezzo giorno, che già erano già pimes (ubriachi) $)$ ), ver. pita s. f. 'sbornia' (Sol. 30), pad., ver. (s)piónsa s. f. 'sbornia, ubriacatura' (Cir. 238, Andr. 418, Sol. 37). Cfr. Wagner 
BF 317 per il port. gerg. piela. Ed ancora Uhlik 25 (serbo-croato) e Kostov 91 (tureo).

$* * *$

La ricognizione di quanto di zingarico si possa rintracciare nei nostri gerghi presenta difficoltà non indifferenti per diversi motivi, che Tagl.Men. hanno posto in evidenza: la mancanza, per esempio, di un repertorio sistematico dello zingaro italiano, che superi la modesta messe offerta fino ad oggi dagli elenchi del Colocci; la possibilità, poi, che attestazioni isolate siano testimonianza piuttosto di puri elementi zingarici, anziché di prestiti assimilati; la facilità di scambiare, con accostamenti poco cauti, per zingarico quello che, a guardar meglio, ha tutt'altra origine (il Wagner VR ha potuto facilmente smontare alcune ricostruzioni del Pasquali, dimostrando l'erronea derivazione zingaresca di almeno sei delle quattordici voci dichiarate di origine zingara); la relativa povertà di indagini puntuali e circoscritte sulle nostre parlate gergali.

Per superare tali difficoltà obiettive è necessario ricorrere a criteri metodologici tali da garantire una sufficiente attendibilità alle proposte: per esempio, al criterio interno delle caratteristiche formali e/o semantiche od a quello esterno della diffusione in altri gerghi fuori d'Italia, raccomandato come essenziale da Tagl.-Men. $246^{3}$

Tra le caratteristiche formali possiamo notare subito alcune formazioni tipiche proprie dello zingarico, come il suff. -(i)ben/(i)pen, ${ }^{4}$ che nello zingaro d'Italia, come di altre parti di Europa, si presenta senza la consonante finale: esso serve a produrre nomi (astratti) da verbi e so stantivi (Pott I 128, Sampson 75). Si sono già visti i due esempi bolognesi čuribé 'ladro' e stàrebi 'prigione':

13. kàrape s. f. 'rivoltella, pistola': ver. (Andr. 413, Sol. 12) $<$ karepen 'colpo, sparo' (Wolf n. 1311), da kar- 'tirare' (cfr. karamaskari);

14. kùrape s. m. 'processo': pad. (Cald. 193) e ver. (Sol. 14) < kùrepen 'lotta, guerra' (Pott I 135, II 113 e 115, Sampson 163, Wolf n. 1605; kùrape 'guerra' e 'processo' anche in Partisani Lomb.), un astratto da kur- colpire. - Si esclude col Wagner BF 304-305 l'appartenenza del port. fam. corripio, corrupio' movimento rapido', 'grande attività' alla medesima base.

3 Tuttavia, per non appesantire troppo l'apparato dei confronti, limitiamo le citazioni a studi abbastanza recenti, che hanno fatto espresso oggetto di ricerca gli zingarismi penetrati nei singoli gerghi: portoghese (Wagner $\mathrm{BF}$ ), francese (Esnault, Max), serbo-croato (Uhlik), rumeno (Juilland), turco (Kostov), oltre ai classici Triandaphyllidis per il greco e Treimer per il cèco, ed oltre al repertorio Rotwelsch del Wolf.

4 Si sono interessati, nel decorso decennio, di questo produttivo suffisso W. P. Schmid ("Indogerm. Forschungen LXVIII, 1963, pp. 276-283) e K. Kostov ( Münchener Studien zur Sprachwissenschaft 18,1965, pp. 41-51). 
Altro diffuso suffisso zingarico è -eng(her)o, composto di -en, proprio del plur. m. obliquo, e di -gro, suffisso di aggettivo di possesso: nei gerghi italiani è presente, come ha rilevato il Wagner VR 282 e 316, in iachengheri 'fiamminiferi' ( $<$ jak 'fuoco') e pastengheri 'pasta alimentare' (composto ibrido), entrambi nel Mirabella. Si riconoscerà, quindi, l'origine zingarica di:

15. doriengo s. m. 'carabiniere' del crem. (Mont. 403: «mi porto dal C. gridandogli non vedi che è un 'doriengo' e ne stanno per venir altri (carabinieri)» e «non mi da fiducia, mi sembra e non sbaglio è un 'doriengo' $)<$ doriengero (Sampson 86-87, Wolf n. 534), da dori 'corda, laccio'; mancano precisi riscontri gergali, ma l'ambulante intervistato da Cald. ha dato per 'questura' la duplice risposta duréngri e peréngri (v. il numero successivo); doriéngari 'gendarmi' è, del resto, voce diffusa fra gli Zingari d'Italia: cfr. Pellis n. 644 e la relativa nota di G. Prati, e Partisani Lomb.

16. pirengo s. m. 'carabiniere': pad. (pinéngri Cald. 195) e ver. (Sol. 30) < pireng(e)ro (Wolf n. 2438 con parallelo nello zing. ted.), da piro 'piede' (cioè, 'agente a piedi': cfr. Sampson 279, pirangló 'scalzo' in Partisani Piem. e, soprattutto, piréskero 'poliziotto', pl. piréngere, Partisani Lomb (da pir-, per- camminare: comun. Soravia). - Fra i gerghi stranieri si noti il ted. pireskro 'poliziotto' Wolf Rotro. n. 4206, proprio anche del cèco (Treimer 79).

17. sonarengo, somarengo s. m. 'carabiniere': ver. (Sol. 37) da accostarsi al šilingr, šilingerák, šelengere del gergo cèco (Treimer 80 ) ed allo Schellenger e var. di quello ted. (Wolf Rotro. n. 4863) < šelengero, da šelo 'fune', cosicché il corrispondente sheléngoro degli Zingari di Rumelia vale 'colui che fa o vende corde' (Paspati 490, Pott II 231, Sampson 347, Wolf n. 3092).

Tipico dei prestiti dallo zingaro è anche il nesso cons. $+d$ del participio passato (Sampson 92): i verbi di origine zingarica accolti in rumeno presentano quasi sempre questa caratteristica (Tagl-.Men. 250), che si ritrova in alcuni participi dei gerghi padani, oltre che nel citato bologn. stardù 'arrestato' (da confrontarsi col parmig. gerg. stardò 'prigione, segreta' Malaspina IV 435, su cui Salvioni in «Romania» XXXIX, 1910, e con lo štardò 'carcere' del gergo di Guardiagrele: Giammarco 225):

18. čòrdo s. m. 'ladro', di cui si parlerà più avanti (al numero 21);

19. rikàrde, rikardór s. m. 'ricettatore': ver. (Sol. 32 assieme a ricardàr 'dare', come in Andr. 417 rikartàr la pila 'dare il denaro') e crem. (Mont. 346: «Così alle undici o poco più eravamo in possesso dell'attacco che portammo a destinazione da un riccardor (ricettatore) in bresciana e il giorno dopo era a Cremona con grana in berta (soldi in tasca)») < riker'tenere' (Pott II 269, Sampson 313, Wolf n. 2743; in Italia rikardó 'ricettatore' Partisani Piem., assieme a rikaráoa 'tenere', e rikardo Partisani Lomb.; 


\section{Linguistica $X V$}

20. stildo s. m. 'arresto': crem. (Mont. gloss., Mont. 404: «mi disse che il C. era in 'stildo' (arrestato)»), da cui stildare v. 'arrestare' (Mont. 403: «io rispondo tu vuoi andare a Cremona a farti 'stildare' (arrestare)») $<$ štildo 'preso, arrestato' (Wolf n. 3178). - Cfr. la nota di G. Prati al n. 2624 del Pellis, oltre a stildó 'arrestato' fra gli Zingari piemontesi (Partisani Piem.) e stildo 'carcerato, stildo, stàrdo 'preso' fra quelli lombardi (Partisani Lomb.).

Anche l'identità semantica (oltre che formale) fra una voce comune di una parlata zingarica italiana e la voce gergale padana può servire come elemento giustificativo di una probabile derivazione di questa da quella. Si lamentava poc'anzi la scarsità del materiale documentario sullo zingaro d'Italia, ma, scorrendo i risultati del rilievo effettuato nel 1932 dal Pellis fra gli Zingari nella località abruzzese L'Annunziata di Giulianova, integrati con il commento di G. Prati, e le relative osservazioni di S. Partisani, e con il glossario degli Zingari d'Abruzzo del Soravia; ricorrendo ai glossari approntati dallo stesso Partisani sulle parlate zingariche dell'Italia centro-meridionale e, soprattutto, del Piemonte e della Lombardia; e rifacendoci all'inchiesta inedita svolta dalla dott. E. Caldognetto Magno a Padova nel 1964, possiamo arricchire le più antiche raccolte dell'Ascoli e del Colocci e rivendicare l'origine zingarica dei seguenti vocaboli del gergo padano:

21. čòr s. m. 'ladro': pad. (čòrdo 'ladro' e čordare 'rubare' Cald. 190), ver. (Sol. 13, accanto a ciorelista 'ladro', ciordàr 'rubare', ciorèl 'furto') e crem. (ciurèl 'furto' Mont gloss.; a ciòrdo 'a rubare' in Mont. 343: «di giorno sulle rive del Po e alla notte a ciòrdo sulla costa piacentina»), molto diffuso anche in altri gerghi ital. (ciori della camorra, lu ččóra dei commercianti di cavalli di Guardiagrele: Giammarco 223) ed europei (Tagl.-Men. 267; si aggiunga per il franc. Max 16, per il ted. Wolf Rotro. n. 5947a, per il serbo-croato Uhlik 12, per il rum. Juilland 162) $<$ čor 'ladro' (Pott II 200, Paspati 538, Sampson 69, Wolf n. 3517 con deriv.); čoraoa 'rubare' ha anche Partisani Merid., čor 'ladro' Soravia Abr., čor 'ladro', čoribén 'furto', čorába 'rubare' Partisani Piem., čor 'ladro' e čórape 'furto' Partisani Lomb.;

22. drum s. m. 'strada': ver. (trón 'luogo, posto, via, appuntamento' Sol. 40) e crem. (Mont. gloss.); cfr. il passaggio di significato in Mirabella: drum 'piano nobile' (su cui Wagner VR 306) e la duplice risposta del gergo ted. Drom e Trom (Wolf Rotro. n. 1097) < drom 'strada, via' (Sampson 89, Wolf $\mathrm{n}$. 555), molto noto anche in Italia: $u$-drome per il Pellis n. 1502 (e G. Prati) e per Soravia Abr., drom in Partisani Merid., Piem. e Lomb.

23. ğòva s. m. pl. 'pidocchi': pad. (Miss. 30, Cald. 191, che ha raccolto anche il completamento svisante gòna fortuna ed anche fmama, ké gé gòoa la fortuna 'stai alla larga, perché quello ha i pidocchi'; era, altresì, allusivo canticchiare il verso della Bohème pucciniana: Cercar che giova...); probabilmente riflesso anche in la gguvanna 'i pidocchi' dei 
mercanti di cavalli di Guardiagrele (Giammarco 224); fuori d'Italia lo ritroviamo, oltre che in Grecia (Triandaphyllidis 16), in Germania (Wolf Rotro. n. 5170) e in Cecoslovacchia (Treimer 76) < ğuo 'pidocchio' (Pott II 214, Paspati 229, Sampson 128, Wolf n. 575); tra gli Zingari italiani è stato segnalato a L'Annunziata (ğubà Pellis trad. n. 34), in Italia meridionale (ğ $u$, pl. ğuà: Partisani Merid.) e settentrionale (ğu: Partisani Piem., ğu, pl. ğua: Partisani Lomb., ğua: Cald. Padova);

24. gra/ni s. m. 'cavallo': pad. (Cald. 191), conosciuto anche da altri gerghi (grassini a Guardiagrele: Pellis 80, lu krasanì per Giammarco 224; poi in ted.: Wolf Rotro. n. 1913, serbo-croato: Uhlik 16, greco: Triandaphyllidis 9, rumeno: Juilland 166; cfr. Wagner VR 310) < grasni 'cavalla' (Pott II 143, Paspati 250, Sampson 111, Wolf n. 936); in Italia: Partisani Merid., Pellis e Soravia Abr., Partisani Piem. e Lomb.;

25. grumie s.f. pl. 'bue, mucca; bovino in genere': pad. (Cald. 192), come nei gerghi tedeschi (Wolf Rotro. n. 1983), serbo-croati (gurumiška: Uhlik 16) e, forse, nel cèco kufr 'vitello' (Treimer 78) < gurumnì 'mucca' (Pott II 141, Paspati 252, Sampson 114, Wolf n. 976; cfr. anche Wagner VR 311); per l'Italia: guroinì 'mucca' nel Sud (Partisani Merid.) e gurùd 'bue' in Soravia Abr., gurumnì 'mucca' in Partisani Piem. e gùrumi, gùrambi 'mucca' in Partisani Lomb.;

26. lači agg. 'bello, buono, bravo': pad. (Cald. 193: 'ladro bravo nel suo lavoro'); cfr. laččó nel gergo dei mercanti di cavalli di Guardiagrele (Giammarco 224) e, fuori d'Italia, isolato nel gergo ted. (Wolf Rotro. $\mathbf{n}$. 3056), serbo-croato (Uhlik 21) e turco (Kostor 91) < lačó 'buono' (Pott II 329, Paspati 328, Sampson 189, Wolf n. 1717); lači in Pellis, lačé, lačó in Partisani Merid., Soravia Abr., Partisani Piem, e Lomb., lačo, lači in Cald. Padooa. - Sarebbe da chiedersi se, in presenza di frasi del tipo marie si-lačì 'Maria è buona' (Pellis trad. n. 24), il ver. cilacio, silacio 'falso, scadente', 'oro falso' (Sol. 13 e 36) non sia un fraintendimento o una voluta antifrasi; ma l'uso in vari dialetti di čilačó 'cattivo' toglie ogni dubbio all'identificazione (comun. Soravia);

27. mangèl (andàr a -) locuz. 'andare a elemosinare': pad. (Cir. 232) e ver. (Andr. 415, Sol. 23 assieme a manghelista 'accattone'; secondo Andr. andàr a mangèl 'mendicare' è passato anche nel dialetto); fuori d'Italia conoscono mangli 'mendicare' il gergo rum. (Juilland 167), diffusamente il gergo ted. (Wolf Rotro. n. 3392) e cèco (Treimer 75 e 78) e, indirettamente, anche quello fr. (Esnault 140, Max 17); il serbo-croato ha mangisati (Uhlik 22), mentre in turco e bulgaro, attraverso uno slittamento semantico, si è giunti all'accezione di 'denaro' (Kostov 89) < mang'mendicare' (Pott II 445, Paspati 348, Sampson 210, Wolf n. 1868); cfr. mangàna 'domandare (per avere)' in Partisani Merid., Piem. e Lomb., ma espressamente 'chiedere l'elemosina' in Soravia $A b r$. ed anche in Partisani Piem. e Lomb.: qui anche mangél 'questua';

28. mui s.f. 'faccia, viso, volto, muso': ver. (Sol. 25), noto anche ai gerghi franc. (ma col significato di 'ventre': Esnault 140), ted. (Wolf 
Rotro. n. 3717), cèco (Treimer 78) e rum. (Juilland 169: mui, moi, muie, màie 'bocca') < mui 'bocca' (Pott II 435, Paspati 368, Sampson 228, Wolf n. 2047), diffusissimo anche fra gli Zingari italiani: in Abruzzo u-mùgé, o'-mùje 'viso' (Pellis n. 128), 'bocca' (Pellis n. 161: ma sospetto è l'ulteriore significato di 'guancia' al n. 155, cfr. G. Prati e Soravia $A b r$.), in Italia meridionale (mù(i) 'bocca', 'faccia': Partisani Merid.) e settentrionale (Partisani Piem. e Lomb.);

29. ràkolo s. m. 'ragazzino, bambino': ver. (Sol. 31); nel pad., in un idiogergo intriso di molte voce zingariche, raklie 'bella ragazza' (Cald.); anche nel gergo franc. (Max 17), al femm. in quello serbo-croato (Uhlik 26), in greco (Triandaphyllidis 14), oltre che in ted. (Wolf Rotro. n. 4473) $\mathrm{e}$ in cèco (Treimer 79) < rakló 'ragazzo' (Pott II 269, Paspati 454, Sampson 306, Wolf n. 2673); la Caldognetto ha raccolto a Padova raklo 'fidanzato', mentre per gli Zingari del sud rakló è il 'ragazzo non zingaro' (Partisani Merid., Soravia Abr.), come in Partisani Piem. e Lomb. (qui ràklo);

30. rada s. f. 'bicicletta': pad. (Cald. 195) e ver. (Sol. 31) < rada 'ruota' (e, nello zing. norveg. rattan 'bicicletta'), registrato nel solo Wolf n. 2661 (ma pure nel Sinto Eftavagarja: comunic. Soravia), per cui si potrebbe pensare anche ad un tedeschismo accattato direttamente dal gergo; cfr., comunque, rada 'ruota' (Partisani Piem. e Lomb.), radéskeri 'bicicletta' (Partisani Lomb.);

31. rati s. f. 'notte': pad. (Cir. 235: 'sera' e sta rati 'questa sera', Cald. Aggiunte), ver. (Sol. 38: starati 'questa sera') e crem. (Mont. gloss.), diffusa anche in altri gerghi (per es., in ted.: Wolf Rotro. n. 4496, cèco: Treimer 79, greco: Triandaphyllidis 14) < rat 'notte' (Pott II 273, Paspati 456, Wolf n. 2698; cfr. anche Pasquali e la relativa chiosa del Wagner); in Italia: ràtte nel Pellis, rat in Partisani Merid. e Soravia Abr., ratì in Partisani Piem. e ràt( $(i)$ in Partisani Lomb.;

32. rùmini $\mathrm{s.f.} \mathrm{'amante,} \mathrm{mantenuta,} \mathrm{donna':} \mathrm{ver.} \mathrm{(Sol.} \mathrm{32),} \mathrm{come} \mathrm{nel}$ gergo franc. (Max 18), serbo-croato (Uhlik 26), cèco (Treimer 79) < romni 'donna' (Pott II 275, Paspati 463, Sampson 318, Wolf n. 2781), notissima anche fra gli Zingari d'Italia: romrì, romnì 'donna (zingara)', 'moglie' (Partisani Merid., Soravia Abr., Partisani Piem. e Lomb.);

33. sàster s. m. 'scalpello': crem. (Mont. gloss.; paretimologicamente potrebbe ritornare nel pad. santi 'scalpelli, strumenti per scardinare' Cir. 236, come nel gergo bologn.: Menarini 121); diffuso nei gerghi ted. nel senso di 'ferro', ma anche in quello di 'scalpello' (Settor, Satro) < saster 'ferro' (Pott II 224, Paspati, Sampson 327, Wolf n. 2856) e con questo significato è noto anche alle parlate zingariche italiane: Pellis trad. n. 37 (sàštre), Partisani Merid. e Soravia Abr. (sáštr(o)), Partisani Piem. (sáster) e Lomb. (sástar);

34. Jdrapa s. f. 'sigaretta': ver. (Sol. 35); nel gergo rum. drap 'tabacco' (Juilland 164) < drab, drap 'erba', 'medicina', 'veleno', 'tabacco' 
(Pott II 316, Paspati 215, Sampson 88, Wolf n. 546); in Italia: drab 'veleno' (Partisani Piem.), 'medicina' (Partisani Lomb.);

35. sero s. m., serùmola s. f. 'capo, testa': ver. (Sol. 35), come anche nel gergo serbo-croato (Uhlik 27) < šeró 'testa' (Pott II 221, Paspati 490, Sampson 347, Wolf n. 3096); in Italia: Pellis, Partisani Merid., Soravia $A b r$., Partisani Piem. e Lomb. (solo qui parola piana: šéro);

36. Jminči s. f. 'donna, ragazza', 'natura': ver. (Sol. 36); tra i gerghi europei l'accolgono il franc. (Max 17, ma Esnault 136 non è dell'avviso di porre sotto questa base altre varianti), il ted. (Wolf Rotro. n. 3615), il cèco (Treimer 78), il serbo-croato (Uhlik 24) e il rumeno (Juilland 168) < minğ 'cunnus' (Pott II 95, Paspati 363, Sampson 222, Wolf n. 1971), raccolta anche da Partisani Merid., Soravia Abr., Partisani Piem. e Lomb. e Caldognetto a Padova;

37. sùkari agg. 'bello, buono, dolce, piacevole': ver. (Sol. 38), cfr. il rum. gerg. șucar e varianti con elevato polisemantismo (Juilland 174176); anche nel gergo serbo-croato (Uhlik 27) e turco (Kostov 90) < šukar 'bello, buono' (Pott II 223, Paspati 485, Sampson 352, Wolf n. 3200); per l'Italia: Partisani Merid., Soravia Abr., Partisani Piem. e Lomb.;

38. tuàkka s.f. 'sigaretta': pad. (Cald. 199) < tubaka 'tabacco' (Wolf n. 3333), in uso anche presso gli Zingari lombardi (tupàka: Partisani Lomb.) e veneti (toà ka 'tabacco': Cald.). ${ }^{5}$

$$
* * *
$$

Dal punto di vista semantico vale la pena di notare la ristrettezza dei campi d'influsso zingarico: $i$ prestiti sono tutti legati ad un modo di vita limitato, nel quale hanno grande parte i piccoli delitti (čor, čordo 'ladro', rikàrde 'ricettatore'), le armi e gli arnesi per effettuarli (karamàskari, kàrape 'rivoltella', čuri 'coltello', sàster 'scalpello' ed anche rada 'bicicletta'), i naturali antagonisti (doriengo, pirengro, sonarengo 'carabiniere') e le naturali conseguenze (stildo 'arresto', stàrdelo 'prendilo', kùrape 'processo', starape 'carcere'). Poi c'è un piccolo squarcio del mondo degli Zingari, dalle persone in (sinto 'zingaro', rùmini 'zingara') e out (čai, gaio 'contadino', ràkolo 'ragazzo') agli animali (grai, gra/ni 'cavallo', čukke 'cane', kakaña 'gallina', grùmie 'bovino'), dalle parti del corpo (mui 'faccia', sero 'testa' con i gooa 'pidocchi', /minči 'natura') agli alimenti (maròko 'pane' ed anche fdrapa, tuàkka 'sigaretta'), ai luoghi,

5 Anche nel gergo carcerario toacca = 'tabacco' (cit. da R. Baccetti Poli n. 156). L'isolamento della voce zingarica è dovuto alla sua penetrazione non antica dal tedesco, come è avvenuto in tanti altri casi (Wagner VR 298-299 cita, per lo zingaro italiano, glas(o) 'bicchiere', flinta 'fucile', strimpi 'calzette', kilma 'mercato', monito 'mese' e non poche altre), ma il tramite zingarico (e non dialettale, attraverso le parlate di confine, come sarebbe stato pur possibile: cfr. le osservazioni di C. Tagliavini a p. 147 della sua recensione a Wagner VR) sembra assicurata dalla identità di forma tra la voce zingara e quella gergale ed inoltre dalla mancanza di riscontri nei dialetti settentrionali. Del resto. elementi germanici sono tanto frequenti nello zingaro italiano, quanto nel gergo degli ambulanti. 


\section{Linguistica $X V$}

momenti ed azioni consuete (pì̀la 'osteria', drum 'strada', rati 'notte', mangèl 'elemosinare'); infine due aggettivi di valore positivo: lači e sùkari, che rappresentano tutto ciò che vi può essere di 'bello, buono, eccellente'.

Come centro di diffusione dobbiamo pensare alla Lombardia non solo per ovvie ragioni geografiche, ma anche per precise considerazioni linguistiche: lo zingarico lombardo presenta spesso, e isolato nel complesso delle parlate italiane e non, un accento retratto, che è riprodotto nei prestiti gergali padani.

Questa semplice escursione, esplorativa e non esauriente, conferma quanto già si riusciva a intuire: i rapporti fra malavita e Zingari, per quanto deboli e sospetti per i motivi chiaramente esposti dal Menarini (Gerghi bolognesi p.14), non potevano non lasciare alcuni precisi residui linguistici. $\mathrm{E}$ certo altre tracce sarà possibile recuperare, quando si potrà, finalmente, disporre del desiderato ampio dizionario dello zingaro italiano.

\section{BIBLIOGRAFIA}

AGNO

ANDR.

ASCOLI

CALD.

CIR.

COLOCCI

ESNAULT
Agno, Il gergo dei portellati, «Gazzetta di Venezia», 2 ottobre 1942 [cfr. R. Baccetti Paoli, Saggio di una bibliografia dei gerghi italiani, Padova, 1953, n. 3].

R. Andrioli, Il dialetto moderno della città di Verona, Padova, 1945-46 [tesi di laurea inedita, discussa col prof. C. Tagliavini; da p. 411 a p. 420: Brede saggio di voci gergali veronesi].

G. I. Ascoli, Zigeunerisches, Halle, 1865.

E. Caldognetto, Contributo ad un dizionario gergale italiano (Le armi), Padova, 1963-64 [tesi di laurea inedita discussa col prof. C. Tagliavini; da p. 181 a p. 215: Appendice. Inchieste gergali dirette: 1. Gergo del Portello (quartiere di Padova); 2. Gergo della leggera (ambulante vissuto tre anni con Zingari); 3. Gergo carcerario (malavita milanese e lombarda); 4. Gergo della malavita triestina; 5. Gergo carcerario di Colle Triglio (Cosenza); 6. Gergo del carcere Castello Aragonese (Castrooillari, prov. di Cosenza)].

O. Ciralli, Il dialetto moderno della città di Padoba, Padova, 1945-46 [tesi di laurea inedita, discussa col. prof. C. Tagliavini; da p. 225 a p. 240: Gergo del Portello].

A. Colocci, Gli Zingari. Storia di un popolo errante, Torino, 1889. [pp. 357-378: Appendice I - Alcune voci del dialetto zingaresco italiano (marchigiano); pp. $379-$ 419: Appendice II - Lessico italiano-tchinghianè. Un controllo eseguito dal Wolf dimostrerebbe, contro i sospetti del Wagner VR, che i dati raccolti sono sostanzialmente attendibili].

G. Esnault, Ciganismes en français et gallicismes des ciganes, "Journal of the Gypsy Lore Society» XIV (1935) $72-86$ e 127-148. 
GIAMMARCO

JUILLAND

KOSTOV

MAX

MONT.

MIRABELLA

MISS.

PARTISANI Lomb.

PARTISANI Merid.

PARTISANI Piem.

PASPATI

PASQUALI

PELLIS

POTT

PRATI G.

SAMPSON

SOL.

SORAVIA $A b r$.
E. Giammarco, I gerghi di mestiere in Abruzzo, «Abruzzo. Rivista dell'Istituto di Studi Abruzzesi» II (1964) 219-239 [ da p. 222 a p. 228: Gergo dei commercianti di cavalli di Guardiagrele (Chieti)].

A. Juilland, Le vocabulaire argotique roumain d'origine tsigane, «Cahiers Sextil Puşcariu» I (1952) 151-181.

K. Kostov, Lehnroörter zigeunerischen Ursprungs im türkischen Argot, «Linguistique Balkanique» XIV, 2 (1970) 83-97.

F. Max, Apports tsiganes dans l'argot français moderne, «Etudes Tsiganes» XVIII, 1 (1972) $12-18$.

D. Montaldi, Autobiografie della leggera, Torino, 1961 ['storie di vita' scritte o dettate da non assimilati della provincia di Cremona; da p. 261 e p. 263: Parole in gergo della malabita in diversi paesi d'Italia (abbrev.: gloss.), predisposto da uno dei protagonisti].

E. Mirabella, Mala vita. Gergo, camorra e costumi degli affiliati con 4500 ooci della lingua furbesca in ordine alfabetico, Napoli, 1910.

B. Missaglia, Anca un fia' de portelato, «Gazzetta Veneta-Seras, 19 maggio 1947 jefr. R. Baccetti Poli n. 468; si cita da una successiva edizione separata [.

S. Partisani, Glossario del dialetto zingaro lombardo, «Lacio Drom IX, 4 (1973) 2-29.

S. Partisani, Glossario degli Zingari dell'Italia CentroMeridionale, «Lacio Drom».VIII, 1 (1972) 2-27.

S. Partisani, Glossario del dialetto zingaro piemontese, «Lacio Drom» VIII, 6 (1972) 11-32.

A. G. Paspati, Etudes sur les Tchinghianés ou Bohémiens de l'Empire Ottoman, Constantinople, 1870.

P. S. Pasquali, Romani Words in Italian Slangs, «Journal of the Gypsy Lore Society», Third Series, XIV (1935) 44-51.

U. Pellis, Il rilieoo zingaresco a L'Annunziata di Giulianopa (Teramo), «Bollettino dell'Atlante Linguistico Italianos II (1936) 61-85.

A. F. Pott, Die Zigeuner in Europa und Asien. Ethnographisch-linguistische Untersuchung, vornehmlich ihrer Herkunft und Sprache, Halle, 1844-1845.

G. Prati, Annotazioni al «Riliedo zingaresco» del Pellis, «Lacio Drom» X, 2 (1974) 26-32, X, 3-4 (1974) 46-57, $\mathrm{X}, 5-6(1974)$ 42-44 [ib., XI, 1-2 (1975) 45-49: S. Partisani, Ossernazioni sulle $\&$ Annotazioni di Guido Prati $\mid$.

$\mathrm{J}$. Sampson, The Dialect of the Gypsies of Wales being the older Form of British Romani preserved in the Speech of the Clan of Abram Wood, Oxford, 1926.

G. Solinas, Glossario del gergo della malavita oeronese. Quaderni di vita veronese, n. 27-30, Verona, 1950.

G. Soravia, Glossario degli Zingari d'Abruzzo, «Lacio Drom» VII, 1 (1971) 2-13. 
SORAVIA Ferr.

TAGL.-MEN.

TREIMER

TRIANDAPHYLLIDIS M. A. Triandaphyllidis, Eine zigeunerisch-griechische Geheimsprache, «Zeitschrift für vergleichende Sprachforschung» LII (1924) 1-42.

UHLIK

WAGNER BF

WAGNER VR

WOLF

WOLFRotro.

G. Soravia, Recensione a E. Ferrero, I gerghi della malavita dal'500 a oggi, Milano, 1972, «Lacio Drom» VIII, 3-4 (1972) 89-92.

C. Tagliavini-A. Menarini, Voci zingare nel gergo bolognese, «Archivum Romanicum» XXII (1938) 242-280 [recensito, fra gli altri, da M. L. Wagner in $>$ Zeitschrift für romanissche Philologie LXI (1941) 363-370; cfr. le riserve espresse da Soravia Ferr.]

K. Treimer, Das tschechische Rotroelsch. Entstehung und Schichten, Heidelberg 1937 [sono dedicate all'elemento zingarico le pp. 74-81].

R. Uhlik, Ciganizmi $u$ šatrooačkom argou $i$ u sličnim gooorima, «Glasnik Zemaljskog Muzeja u Sarajevu. Istorija i etnografija» N. s., IX (1954) 5-31.

M. L. Wagner, $O$ elemento cigano no calão e na linguagem popular portuguesa, $\star$ Boletim de filologia» $\mathrm{X}$ (1949) 296-319.

M. L. Wagner, Ubersicht über neuere Veröffentlichungen über italienische Sondersprachen. Deren zigeunerische Bestandteile, «Vox Romanica» I (1936) 264-317 [integrabile con le recensioni di C. Tagliavini in $\gg$ Archivum Romanicum* XXII (1938) 145-148 e U.Pellis in «Bollettino dell'Atlante Linguistico Italiano» II (1936) 9092].

S. A. Wolf, Grosses Wörterbuch der Zigeunersprache (romani tšin). Wortschatz deutscher und anderer europäischer Zigeunerdialekte, Mannheim, 1960.

S. A. Wolf, Wörterbuch des Rotrolschen. Deutsche Gaunersprache, Mannheim, 1956.

\section{Poozetek}

\section{CIGANSKE BESEDE V ARGOJU PADSKE NIŽINE}

Zanimanje za ciganski jezik je staro. Od slovenskih jezikoslovcev se je s ciganskimi govori temeljito ukvarjal že Miklošič $\nabla$ delu Ưber die Mundarten und die Wanderung der Zigeuner Europa's.

Avtor analizira vdor ciganskih besedi $\vee$ argoje mest in vasi pokrajine okrog Padove in navaja skoro štirideset besedí, ki so po vsej verjetnosti ciganskega izvora. Za semantiko je zanimivo, da so besede zajete $v$ samo nekaj semantičnih polj, in sicer zaradi načina življenja, kot npr. semantično polje majhnih tatvin, orožja in sredstev za take tatvine (izrazi za tatu, za shranjevanje ukradenega blaga, za samokres, nož, kolo), za naravne nasprotnike (karabiner) in za samo po sebi umerne posledice take dejavnosti (aretacije, proces, zapor). Semantično polje predstavlja tudi ciganski domači svet (cigan, otrok, konj, pes, govedo, kokoš), deli telesa, hrana, cigarete. Studija potrjuje, da so zveze med »podzemljem « in ciganskim življenjem dale nekaj pomensko zanimivih izposoj. 